\title{
Therapeutic Drug Monitoring and Impact Indicator of Vancomycin Pharmacokinetics in Abdominal Cancer Patients complicated with Severe Infectious Disease
}

Xiaowu Zhang ( $\square$ zhangxiaowu1986@163.com )

Tianjin medical University Cancer Institute and Hospital https://orcid.org/0000-0002-3751-6348

Yang Lyu

Tianjin Medical University Cancer Institute and Hospital

Donghao Wang

Tianjin Medical University Cancer Institute and Hospital

Research article

Keywords: Vancomycin; Abdominal cancer; Trough concentration; Pharmacokinetics; Severe infectious

Posted Date: May 9th, 2019

DOI: https://doi.org/10.21203/rs.2.9533/v1

License: (a) (1) This work is licensed under a Creative Commons Attribution 4.0 International License. Read

Full License 


\section{Abstract}

Abstract Objective: This study was designed to investigate effectiveness of therapeutic drug monitoring (TDM) and impact indicator of vancomycin pharmacokinetics in abdominal cancer patients complicated with severe infectious disease. Methods: A total of 78 patients abdominal cancer patients complicated with severe infectious disease were included. Vancomycin serum trough concentrations were measured using the fluorescence polarization immunoassay (FPIA) method. The patients were divided into early and delayed groups based on whether they achieve the target concentration. And clinical factors were compared between two groups. Results: The average initial therapeutic dose of vancomycin was $15.18 \pm 3.29 \mathrm{mg} / \mathrm{kg}$ (q12h). Ultimately, we collected 78 patient's trough concentrations data. The research revealed that the abdominal cancer patients complicated with severe infectious disease had significantly lower initial vancomycin trough concentrations (median [IQR]: $6.90[5.28-11.20] \mathrm{mg} / \mathrm{L}$ ) compared with the recommended standard goal vancomycin trough concentration (10-15 or $15-20 \mathrm{mg} / \mathrm{L}$ ). Multiple regression analysis revealed that Cys-C was the most important variable for vancomycin target trough achievement. We divided patients into early and delayed groups based on whether the initial trough concentration achieved standard goal trough concentration. Although the clinical outcomes were similar between two groups, the duration of mechanical ventilation in Early group was considerably shorter compared with group Delayed group $(x 2=4.532 ; p<0.05$; Fig 1E). Propensity score weighting further confirmed that the duration of mechanical ventilation $(X 2=6.607 ; p$ $<0.05$; Fig $1 F)$ and vasoactive agent $(X 2=6.106 ; p<0.05$; Fig 1D) was considerably shorter compared with

group Delayed group. Conclusions: The steady-state initial vancomycin trough concentration was significantly reduced in abdominal cancer patients complicated with severe infectious disease. Higher initial dosage regimen is needed to ensure clinical effectiveness. The baseline Cys-C level measured prior to administration of vancomycin is suggested to be the most suitable parameter to predict whether vancomycin trough concentration is up to standard dosage. Early attainment of target concentration significantly improved hemodynamic stability and reduced duration of mechanical ventilation.

\section{Background}

Vancomycin is a bactericidal glycopeptide antibiotic which inhibits bacterial growth by hindering the synthesis of cell wall in bacteria. It exerts strong antibiotic effect to Gram-positive bacteria. It includes methicillinresistant Staphylococcus aureus (MRSA), Enterococcus, or methicillin-resistant Staphylococcus epidermidis (MRSE) [1]. The pharmacokinetic-pharmacodynamic breakpoint of vancomycin is defined as the ratio of the area under the concentration-time curve from 0 to 24 hours (area under the curve (AUC) 0-24) to minimal inhibitory concentration (MIC), and is at least $400 \mathrm{~h}$ in adults with Staphylococcus aureus pneumonia [2]. Intravenous vancomycin mainly combined with albumin and IgA, protein-bound protein is $25 \%$ to $50 \%$, almost completely eliminated by the renal pathway. Therefore, the most important factor in determining vancomycin dosage is renal function [3]. Therapeutic drug monitoring (TDM) as an optimizing vancomycin therapy is widely recommended for avoiding secondary clinical complications because of its narrow therapeutic window, such as vancomycin toxicity due to over-dosing or resistance due to under-dosing [3].

Patients with malignant tumors represent a critical population in whom inadequate empirical antibacterial therapy may lead to increased infection-related morbidity and mortality. In addition, previous studies have confirmed that pharmacokinetic parameters often exhibit different characteristics than patients with non- 
cancer diseases [4] [5], making optimization of drug dosing regimen essential. Research confirms that vancomycin has an increased clearance rate in adults with malignant hematological disease compared with adults without malignant tumors, but the pharmacokinetic data are limited [6] [7].Therefore, we aimed to clarify the possible impact of this difference in pharmacokinetic on the routine vancomycin therapy and conducted a retrospective study on the factors influencing the trough concentrations for vancomycin dose adjustment in abdominal cancer patients complicated with severe infectious disease.

\section{Methods}

Subjects: Between February 2014 to June 2017, we retrospectively reviewed 78 patients of abdominal cancer aged more than 18 years who were complicated with severe infectious disease and who were treated in the intensive care unit of Tianjin Cancer Hospital (Tianjin, China). This clinical trial was agreed by the Ethics Committee and approval from the Ethics Committee of Tianjin Medical University Cancer Institute and Hospital and complied with the Declaration of Helsinki. And their data were collected from the clinical charts. All patients received vancomycin therapy for more than five estimated terminal disposition half-lives, by which time serum vancomycin levels were supposed to have reached a steady state. Human subjects with poor kidney function ( $\mathrm{CLCr}<50 \mathrm{~mL} / \mathrm{min}$ ), pregnant and lactating women, hemodialysis patients were excluded. Other anti-infective treatments were administered if patient condition needs.

Pharmacokinetic analysis and Vancomycin therapeutic drug monitoring (TDM):

The vancomycin dosage was administered over a 1-hour period. The steady state vancomycin trough concentration was measured prior to subsequent treatment to adjust dose and dose intervals [8] . The target serum vancomycin trough concentrations ranged from $10-15$ or $15-20 \mathrm{mg} / \mathrm{L}[9]$.Serum vancomycin concentrations were measured by FPIA method using a Cobas 6000 c501 analyzer (Roche Diagnostics, China). Meanwhile, the laboratory staff records information about each specimen, including gender, age, body weight, serum creatinine concentration, daily dosage, dose interval, infusion time, sampling time since infusion end and measure concentration for pharmacokinetic analysis.

Data collection:

Demographic data obtained included age, gender, admission diagnosis, acute physiology and chronic health assessment II score (Apache II score) at admission to the ICU. For the treatment program, daily doses, interval time, and the occurrence of acute kidney injury (AKI) and renal replacement therapy (RRT) were recorded. Newonset acute kidney injury was defined according to the KDIGO (Kidney Disease: Improving Global Outcomes) stage II criteria after at least 24 hours and within 7 days of vancomycin administration initiation [10]. The duration of the vasoactive agent, the duration of mechanical ventilation, the duration of the antibiotic, and the 28-day all-cause mortality were also recorded. The definition of clinical outcomes after cessation of the study drug, including clinical success and clinical failure, are shown in Table 1. Finally, inverse probability of treatment weighting (IPTW) was used to measure participants based on the estimated exposure probability (the propensity score) for a given confounding factor to balance the observed confounding factors between the early and delayed groups.

Statistical analysis 
Values for categorical variables are given as count (percentage), for continuous variables, as mean \pm standard deviation or as median [interquartile range]. Pearson $c^{2}$ test was used for categorical variables, and $t$ test was used for continuous variables. Univariate and multivariate analysis is used for covariates associated with target trough achievement. Survival was estimated by the Kaplan-Meier method and compared using the logrank test. All statistical analyses were performed using the SPSS statistical package (version 24.0, SPSS Inc., Chicago), $P \otimes 0.05$ was considered statistically significant.

\section{Results}

A total of 78 patients received recommended standard vancomycin dosage adjustment. Clinical characteristics, pharmacokinetic parameters and clinical outcomes are summarized in Table 2 and Table 3. Our research revealed that the abdominal cancer patients complicated with severe infectious disease have a significantly lower initial vancomycin trough concentration (median [IQR]: 6.90[5.28-11.20] mg/L) than the recommended standard vancomycin trough concentrations (10-15 or 15-20 mg/L). The overall relationship between trough concentrations and potential covariates was screened by Univariate and multivariate analysis to explore potential information covariates. There was a strong correlation between vancomycin trough concentration and age, body weight, serum creatinine and serum Cystatin C level (Cys-C) (Table 4). Multivariate regression analysis revealed that the Cys-C was the most important variable for vancomycin target trough achievement (odds ratio, $5.274 ; 95 \% \mathrm{Cl}, 1.780$ to $15.627 ; p=0.003$ ) (Table 4).

We divided patients into Early group and Delayed group based on whether the initial trough concentration achieved the target concentration. Although the Clinical outcomes were similar between two groups in Table 5 (e. g. , the incidence of new-onset AKI or RRT, clinical success rate, 28-day all-cause mortality), the duration of mechanical ventilation in Early group was considerably shorter compared with Delayed group $\left(\chi^{2}=4.532 ; p<\right.$ 0.05 ;Fig 1E). Propensity score weighting (IPTW) further confirmed that the duration of mechanical ventilation $\left(X^{2}=6.607 ; p<0.05 ;\right.$ Fig $\left.1 F\right)$ and vasoactive agent $\left(X^{2}=6.106 ; p<0.05 ;\right.$ Fig 1D) in Early group were considerably shorter compared with Delayed group.

\section{Discussion}

Therapeutic drug monitoring of vancomycin is widely recommended for clinical treatment[11] [9]. However, few studies have been available to evaluate vancomycin (VCM) pharmacokinetics in abdominal cancer patients complicated with severe infectious disease. The aim of this study was to address the above issues and sought to find clinically useful information to predict and estimate the appropriate dosage of vancomycin. This study clearly demonstrated the changes in the pharmacokinetic profile of vancomycin in abdominal cancer patients complicated with severe infectious disease for the first time. The conclusion is that initial vancomycin trough concentrations are significantly reduced in these patients. We also found that Cys- $C$ was associated with target trough achievement. Accordingly, the traditional standard dose of vancomycin may result in a high risk of failing to achieve the recommended standard vancomycin trough concentrations. This finding confirms the need to design useful guidelines for vancomycin dosage individualization in abdominal cancer patients complicated with severe infectious disease. 
Previous studies have shown that malignant tumors themselves may have an impact on VCM PKs [12], in addition to changes in volume of distribution due to edema, peritoneum or pleural effusion. Chang et al. reported an increase in VCM clearance(CL) in pediatric cancer patients using the 2-compartment Bayesian PK approach and this was also confirmed in adult hematological malignancies[13]. On the other hand, there are also different viewpoint, Omote et al. showed no significant differences in estimated VCM clearance between cancer patients and non-cancer patients, suggesting dosage adjustment by routine TDM only [14]. Nevertheless, Shimada et al. proposed a direct activation of the renal organic anion/cation transporters (OCT1/2, OATs) by cytokines such as TNF-a, which indeed increased in the context of cancer in vivo experiments [15] [16].

Severe infectious disease was frequently combined with the development of systemic inflammatory response syndrome (SIRS). This inflammatory response leads to vasodilatation, capillary leakage and the development of hyperdynamic cardiovascular state characterized by third-space, high cardiac output and increased blood flow to the major tissue and organ [17].As water soluble, vancomycin is primarily cleared by the kidneys. Increased CL is very likely due to increase in renal blood flow, which enhances the elimination of vancomycin by urine and leads to a decrease in plasma vancomycin concentration. And increased volume of distribution $(V d)$ is usually attributed to the third space induced by SIRS [18], which results in significant overhydration. However, the underlying mechanism for increasing vancomycin CL and Vd still need further studied.

Recently, augmented renal clearance (ARC) has been proposed to describe the enhancement of renal elimination of circulating solutes observed in critically ill patients [19]. Most studies have shown that hyperdynamic circulation is characterized by increased renal blood flow with an increase in glomerular filtration rate as a potential mechanism [20]. Nevertheless, no information was given concerning the number of oncological patients in these studies. Previous study found that the risk factors for ARC are age, sepsis, and SIRS [19]. Thus, the increased vancomycin CL observed in this study was very likely associated with ARC but

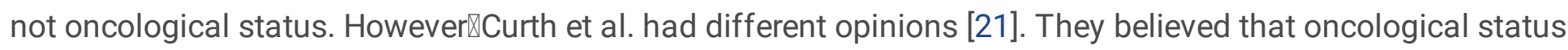
can also cause occurrence of ARC. Considering that the study is only a case report, this opinion should be treated with caution. In practice, there is no guidance for this treatment and ARC has not been taken seriously in clinical work in China. Clinicians usually do not seriously consider increasing the dose even though dose adjustment is allowed at their discretion and the actual therapeutic drug monitoring results showed very low concentrations.

Of these 78 patients, small number of patients achieved the target level. The standard vancomycin dose recommended in the package instructions approved by the Chinese authorities appears to be too low to achieve the target trough concentrations in clinical practice. The reason for this result may be because the standard vancomycin administration algorithm was developed based on data from relatively healthy patients. And our research strongly recommends that abdominal cancer patients require higher dose regimens.

In this research, we observed a strong correlation between vancomycin trough concentrations and age, body weight, serum creatinine and especially the Cys-C. This research confirmed the relationship between Cys-C and vancomycin trough concentrations for the first time in abdominal cancer patients. Cys-C is a non-glycosylated, low molecular weight basic protein containing 120 amino acids [22]. Human Cys-C is a housekeeping gene, and serum Cys-C is stably produced by all human nucleated cells [22]. In earlier studies, Cys-C was assessed 
to be independent of age, muscle mass or body mass index in healthy individuals [23]. Although previous studies have shown a significant correlation between serum Cys-C levels and malignant progression of colorectal cancer, melanoma and ovarian cancer [24], recent studies confirmed that Cys-C can be very effective in predicting renal function in cancer patients [25] [26]. These features partially explain the relationship between Cys- $\mathrm{C}$ and vancomycin trough concentrations.

This observational study was done on a real-time clinical practice. Since the serum Cys-C is readily available in clinical practice, these parameters may allow physicians and/or pharmacists to predict vancomycin dose requirements in a short period of time. Earlier identification of insufficient vancomycin trough concentrations could be used to optimize antimicrobial adequacy. Earlier initial goal vancomycin trough concentration achievement means shorter duration of mechanical ventilation, and shorter duration of vasoactive agent, which means earlier hemodynamic stability. With this conclusion, clinicians should be able to adjust the dose of vancomycin confidently. For specific dose adjustment algorithm, we need further research. Recently, Erin Frazee et al have confirmed that vancomycin dosing algorithm based on estimated glomerular filtration rate from creatinine and Cys-C levels significantly improved goal trough achievement compared to Cockcroft-Gault creatinine clearance among ICU patients with stable kidney function [27].

There are several potential limitations in research. First, the samples collected in this study were too limited to accurately assess the pharmacokinetics of vancomycin. Second, this is single-center and observational study with bias in case selection. Third, Inappropriately timed vancomycin trough concentration determination is a general challenge for therapeutic drug monitoring and is present regardless of the vancomycin administration algorithm used.

\section{Conclusions}

The serum initial trough concentration of vancomycin was significantly reduced in abdominal cancer patients complicated with severe infectious disease. Clinicians should pay special attention to changes in vancomycin pharmacokinetic, and higher dosage regimens are needed to ensure clinical effectiveness. The Cys-C level measured prior to vancomycin was administered is considered to be a potentially valuable parameter for predicting whether the vancomycin trough concentration is up to standard. Further, this conclusion still requires larger studies to confirm these observations, which will assist in establishing guidance of vancomycin dosing algorithm.

\section{Abbreviations}

APACHE II, acute physiology and chronic health evaluation II;

AKI, acute kidney injury;

Cys-C, Cystatin C;

RRT, renal replacement therapy;

SOFA, Sequential Organ Failure Assessment; 
VCM, Vancomycin.

\section{Declarations}

\section{Acknowledgment}

We thank the health professional staff from Department of Clinical Laboratory, Tianjin Medical University Cancer Institute and Hospital,

\section{Ethics approval and consent to participate}

This retrospective cohort study was agreed by the Ethics Committee and approval from the Ethics Committee of Tianjin Medical University Cancer Institute and Hospital and waived the requirement to obtain informed consent

\section{Consent for publication}

Not applicable.

\section{Availability of data and materials}

The datasets used and/or analyzed during the current study are available from the corresponding author on reasonable request.

\section{Competing Interest}

The authors declare that they have no competing interests.

\section{Funding}

The study was supported by a grant from the Oncology and Translational Medicine of Tianjin Medical University Cancer Institute and Hospital, China (No 1611). The funders had no role in study design, data collection, data analysis, and data interpretation or writing of the manuscript.

\section{Authors' Contributions}

Xiaowu Zhang $\mathbb{D}$ Donghao Wang were involved in the concept, interpretation of the data and writing of the manuscript. Yang Lyu was involved in the statistical analyses and the writing of the manuscript.

\section{References}

1. Liu C, Bayer A, Cosgrove SE, Daum RS, Fridkin SK, Gorwitz RJ, Kaplan SL, Karchmer AW, Levine DP, Murray $B E$ et al: Clinical practice guidelines by the infectious diseases society of america for the treatment of methicillin-resistant Staphylococcus aureus infections in adults and children. Clinical infectious diseases : an official publication of the Infectious Diseases Society of America 2011, 52(3):e18-55. 
2. Moise-Broder PA, Forrest A, Birmingham MC, Schentag JJ: Pharmacodynamics of vancomycin and other antimicrobials in patients with Staphylococcus aureus lower respiratory tract infections. Clinical pharmacokinetics 2004, 43(13):925-942.

3. Rybak MJ, Lomaestro BM, Rotschafer JC, Moellering RC, Craig WA, Billeter M, Dalovisio JR, Levine DP: Vancomycin therapeutic guidelines: a summary of consensus recommendations from the infectious diseases Society of America, the American Society of Health-System Pharmacists, and the Society of Infectious Diseases Pharmacists. Clinical infectious diseases : an official publication of the Infectious Diseases Society of America 2009, 49(3):325-327.

4. Bubalo JS, Munar MY, Cherala G, Hayes-Lattin B, Maziarz R: Daptomycin pharmacokinetics in adult oncology patients with neutropenic fever. Antimicrobial agents and chemotherapy 2009, 53(2):428-434.

5. Romano S, Fdez de Gatta MM, Calvo MV, Caballero D, Dominguez-Gil A, Lanao JM: Population pharmacokinetics of amikacin in patients with haematological malignancies. The Journal of antimicrobial chemotherapy 1999, 44(2):235-242.

6. Buelga DS, del Mar Fernandez de Gatta M, Herrera EV, Dominguez-Gil A, Garcia MJ: Population pharmacokinetic analysis of vancomycin in patients with hematological malignancies. Antimicrobial agents and chemotherapy 2005, 49(12):4934-4941.

7. Fernandez de Gatta Mdel M, Santos Buelga D, Sanchez Navarro A, Dominguez-Gil A, Garcia MJ: Vancomycin dosage optimization in patients with malignant haematological disease by pharmacokinetic/pharmacodynamic analysis. Clinical pharmacokinetics 2009, 48(4):273-280.

8. He J, Mao EQ, Feng J, Jiang HT, Yang WH, Chen EZ: The pharmacokinetics of vancomycin in patients with severe acute pancreatitis. European journal of clinical pharmacology 2016, 72(6):697-702.

9. Martin JH, Norris R, Barras M, Roberts J, Morris R, Doogue M, Jones GR: Therapeutic monitoring of vancomycin in adult patients: a consensus review of the American Society of Health-System Pharmacists, the Infectious Diseases Society of America, and the Society Of Infectious Diseases Pharmacists. The Clinical biochemist Reviews 2010, 31(1):21-24.

10. Khwaja A: KDIGO clinical practice guidelines for acute kidney injury. Nephron Clinical practice 2012, 120(4):c179-184.

11. Ye ZK, Chen YL, Chen K, Zhang XL, Du GH, He B, Li DK, Liu YN, Yang KH, Zhang YY et al: Therapeutic drug monitoring of vancomycin: a guideline of the Division of Therapeutic Drug Monitoring, Chinese Pharmacological Society. The Journal of antimicrobial chemotherapy 2016, 71(11):3020-3025.

12. Sadoh S, Tsuji Y, Tsukamoto K: [Correlation of pharmacokinetic parameters with serum vancomycin concentration in elderly patients with malignancies]. Yakugaku zasshi : Journal of the Pharmaceutical Society of Japan 2010, 130(1):69-73.

13. Chang D: Influence of malignancy on the pharmacokinetics of vancomycin in infants and children. The Pediatric infectious disease journal 1995, 14(8):667-673.

14. Omote S, Yano Y, Hashida T, Masuda S, Yano I, Katsura T, Inui K: A retrospective analysis of vancomycin pharmacokinetics in Japanese cancer and non-cancer patients based on routine trough monitoring data. Biological \& pharmaceutical bulletin 2009, 32(1):99-104.

15. Balkwill F: Tumour necrosis factor and cancer. Nature reviews Cancer 2009, 9(5):361-371. 
16. Shimada I, Iwata C, Taga S, Teramachi H, Nomura M, Miyamoto K, Tsuciya H, Wada T, Kimura K, Matsushita R: Enhanced renal clearance of vancomycin in rats with carcinogen-induced osteosarcoma. Anticancer research 2012, 32(3):823-829.

17. Shimamoto Y, Fukuda T, Tanaka K, Komori K, Sadamitsu D: Systemic inflammatory response syndrome criteria and vancomycin dose requirement in patients with sepsis. Intensive care medicine 2013, 39(7):1247-1252.

18. del Mar Fernandez de Gatta Garcia M, Revilla N, Calvo MV, Dominguez-Gil A, Sanchez Navarro A: Pharmacokinetic/pharmacodynamic analysis of vancomycin in ICU patients. Intensive care medicine 2007, 33(2):279-285.

19. Udy AA, Roberts JA, Lipman J: Implications of augmented renal clearance in critically ill patients. Nature reviews Nephrology 2011, 7(9):539-543.

20. He J, Mao E, Jing F, Jiang H, Yang W, Chen E: [Pharmacokinetics of vancomycin in patients with severe acute pancreatitis and its influencing factors: analysis of 7 years data]. Zhonghua wei zhong bing ji jiu yi xue 2017, 29(6):491-495.

21. Curth HM, Pelc A, Kutting F, Steffen HM: Augmented renal vancomycin clearance in cancer patients: a case report and review of the literature. Oncology research and treatment 2015, 38(4):182-184.

22. Randers E, Erlandsen EJ: Serum cystatin $\mathrm{C}$ as an endogenous marker of the renal function-a review. Clinical chemistry and laboratory medicine 1999, 37(4):389-395.

23. Fliser D, Ritz E: Serum cystatin C concentration as a marker of renal dysfunction in the elderly. American journal of kidney diseases : the official journal of the National Kidney Foundation 2001, 37(1):79-83.

24. Kos J, Krasovec M, Cimerman N, Nielsen HJ, Christensen IJ, Brunner N: Cysteine proteinase inhibitors stefin $A$, stefin $B$, and cystatin $C$ in sera from patients with colorectal cancer: relation to prognosis. Clinical cancer research : an official journal of the American Association for Cancer Research 2000, 6(2):505-511.

25. Yang F, Li D, Di Y, Zhang Y, Zang Y, Ren J, Yan L, Zhou Z, Liu H, Xu Z: Pretreatment Serum Cystatin C Levels Predict Renal Function, but Not Tumor Characteristics, in Patients with Prostate Neoplasia. BioMed research international 2017, 2017:7450459.

26. Ohara G, Miyazaki K, Kurishima K, Kagohashi K, Ishikawa H, Satoh H, Hizawa N: Serum levels of cystatin $C$ in elderly lung cancer patients. Oncology letters 2012, 3(2):303-306.

27. Frazee E, Rule AD, Lieske JC, Kashani KB, Barreto JN, Virk A, Kuper PJ, Dierkhising RA, Leung N: Cystatin C-Guided Vancomycin Dosing in Critically III Patients: A Quality Improvement Project. American journal of kidney diseases : the official journal of the National Kidney Foundation 2017, 69(5):658-666.

\section{Tables}

Table 1₫Clinical outcome definitions after study drug cessation 


\begin{tabular}{ll}
\hline Clinical response & \multicolumn{1}{c}{ Definition } \\
\hline Clinical success & $\begin{array}{l}\text { 1. Cure or improvement of all signs and symptoms caused by the infection } \\
\text { 2. No additional antibiotic therapy required }\end{array}$ \\
\hline Clinical failure & $\begin{array}{l}\text { 1. Persistent or worsening of any one of the clinical symptoms } \\
\text { 2. New clinical signs and symptoms of infection }\end{array}$ \\
\hline & 3. Other systemic antimicrobial therapy required \\
\hline
\end{tabular}

Table 2. Vancomycin Levels Achieved and Clinical Outcomes $(n=78)$

\begin{tabular}{lc}
\hline Characteristics & \\
\hline Sex (male/female) & $17 / 61$ \\
\hline Age (years) & $65.04 \pm 12.72$ \\
\hline & $65[57-63]$ \\
\hline Body weight $(\mathrm{kg})$ & $24.74 \pm 3.68$ \\
\hline Apache II score & $79[71.5-86]$ \\
\hline SOFA score & $20.05 \pm 2.92$ \\
\hline & $19[18-22]$ \\
\hline Albumin $(g / L)$ & $13.15 \pm 2.25$ \\
& $13[12-15]$ \\
\hline Serum creatinine $(\mu \mathrm{mol} / \mathrm{L})$ & $27.89 \pm 6.14$ \\
\hline Cys-C $(\mathrm{mg} / \mathrm{L})$ & $28.20[24.85-32.30]$ \\
\hline
\end{tabular}

\section{Cancer type, $\mathbf{n}(\%)$}

Hepatocellular carcinoma 16(20.5)

Pancreatic cancer 6(7.7)

Colorectal cancer 22(28.2)

Gastric cancer 32(41.0)

Gallbladder cancer $\quad 2(2.6 \%)$

\section{Suspected source of infection}

\begin{tabular}{lc} 
Pulmonary & $39(50)$ \\
\hline Intra-abdominal & $28(35.9)$ \\
Surgical incision & $3(3.8)$ \\
\hline Other/unknown & $8(10.3)$
\end{tabular}

\section{Identified pathogens, $\mathbf{n}(\%)$}

\begin{tabular}{ll} 
methicillin-resistant Staphylococcus aureus & $21(26.9)$ \\
methicillin-resistant Staphylococcus epidermidis & $12(15.4)$ \\
\hline Enterococcus & $35(44.9)$ \\
\hline Other Gram-positive coccus & $10(12.8)$ \\
\hline
\end{tabular}

Abbreviations: APACHE II, acute physiology and chronic health evaluation II; SOFA, Sequential Organ Failure Assessment; Cys-C, Cystatin C. 
Table 3. Vancomycin Levels Achieved and Clinical Outcomes $(n=78)$

\begin{tabular}{lc}
\hline Characteristic & \\
\hline Initial dose (daily), $\mathbf{n}(\%)$ & $4(5.1 \square$ \\
\hline $1000-2000 \mathrm{mg}$ & $50(64.1 \square$ \\
\hline $2001-3000 \mathrm{mg}$ & $24(30.8 \square$ \\
\hline$\square 3000 \mathrm{mg}$ & $15.18 \pm 3.29$ \\
\hline Average daily dosage (mg/kg, q12h) & $15.38[14.29-19.27]$ \\
\hline & \\
\hline Vancomycin trough concentration, $\mathbf{n}$ (\%) & $55(70.5)$ \\
\hline$\square 10 \mathrm{mg} / \mathrm{L}$ & $15(19.3)$ \\
\hline $10-15 \mathrm{mg} / \mathrm{L}$ & $8(10.3)$ \\
\hline $15-20 \mathrm{mg} / \mathrm{L}$ & $8.26 \pm 5.01$ \\
\hline Average trough concentration (mg/L) & $6.90[5.28-11.20]$ \\
\hline & $4[2-5]$ \\
\hline Duration of vasoactive agent(day) & $4[3-5]$ \\
\hline Duration of ventilation(day) & $5[5-7]$ \\
\hline Duration of antibiotics(day) & $7(9.0)$ \\
\hline Composite outcome of new-onset AKI or RRT & $58(74.4)$ \\
\hline Clinical success rate, $\mathrm{n}(\%)$ & $4(5.1)$ \\
\hline 28-day all-cause mortality & \\
\hline
\end{tabular}

Abbreviations: AKI, acute kidney injury; RRT, renal replacement therapy.

Table 4. Univariate and Multivariate Analysis of Covariates Associated With Target Trough Achievement

\begin{tabular}{|c|c|c|c|c|c|c|}
\hline \multirow[b]{2}{*}{ Variants } & \multicolumn{3}{|c|}{ Univariate Analysis } & \multicolumn{3}{|c|}{ Multivariate Analysis } \\
\hline & OR & $95 \% \mathrm{CI}$ & $p$ & OR & $95 \% \mathrm{CI}$ & $p$ \\
\hline Sex & 1.470 & $0.423-5.105$ & 0.544 & & & \\
\hline Age (years) & 1.057 & $1.003-1.114$ & $0.039 *$ & 1.152 & $0.997-1.331$ & 0.054 \\
\hline Body weight(kg) & 1.057 & $1.002-1.116$ & $0.043^{*}$ & 0.929 & $0.804-1.073$ & 0.315 \\
\hline Apache II score & 1.016 & $0.863-1.198$ & 0.846 & & & \\
\hline SOFA score & 1.006 & $0.809-1.250$ & 0.959 & & & \\
\hline Albumin $(\mathrm{g} / \mathrm{L})$ & 1.007 & $0.925-1.097$ & 0.864 & & & \\
\hline Serum creatinine $(\mu \mathrm{mol} / \mathrm{L})$ & 1.018 & $1.002-1.035$ & $0.032 *$ & 1.018 & $0.993-1.045$ & 0.158 \\
\hline Cys-C(mg/L) & 5.249 & $1.972-13.972$ & $0.001 *$ & 5.274 & $1.780-15.627$ & $0.003^{*}$ \\
\hline
\end{tabular}

$* p \llbracket 0.05$

Abbreviations: APACHE II, acute physiology and chronic health evaluation II; SOFA, Sequential Organ Failure Assessment; Cys-C, Cystatin C.

Table 5 $\square$ Patients' Baseline Data Before and After Propensity Score Weighting $(n=78)$ 


\begin{tabular}{|c|c|c|c|c|c|c|c|c|}
\hline \multirow[b]{3}{*}{ Characteristics } & \multirow{2}{*}{\multicolumn{2}{|c|}{ Entire Cohort }} & \multirow[b]{3}{*}{$c^{2}$} & \multicolumn{5}{|c|}{ Propensity Score } \\
\hline & & & & & Weig & & & \\
\hline & $\begin{array}{l}\text { Delayed } \\
\text { group }\end{array}$ & Early group & & $P$ & $\begin{array}{l}\text { Delayed } \\
\text { group }\end{array}$ & Early group & $c^{2}$ & $P$ \\
\hline & $(n=55)$ & $(n=23)$ & & & $(n=23)$ & $(n=23)$ & & \\
\hline \multicolumn{9}{|l|}{ Age,years } \\
\hline Mean \pm S.D. & $62.65 \pm 11.87$ & $68.78 \pm 10.45$ & & $0.035^{*}$ & $65.22 \pm 10.76$ & $68.78 \pm 10.45$ & & 0.260 \\
\hline Median(range) & $65(56-70)$ & $68(61-76)$ & & & $67(60-74)$ & $68(61-76)$ & & \\
\hline Sex,n(\%) & & & & 0.542 & & & & 0.381 \\
\hline Male & $42 \square 76.4 \% \square$ & $19 \square 82.6 \% \square$ & & & $21 \llbracket 91.3 \%)$ & $19 \square 82.6 \% \square$ & & \\
\hline Female & 13ロ23.6\%凸 & $4 \square 17.4 \square$ & & & $2(8.7 \%)$ & $4 \square 17.4 \square$ & & \\
\hline \multicolumn{9}{|l|}{$\begin{array}{l}\text { Body } \\
\text { weight,kg }\end{array}$} \\
\hline Mean \pm S.D. & $75.53 \pm 12.38$ & $81.61 \pm 9.302$ & & $0.038 *$ & $78.52 \pm 11.63$ & $81.61 \pm 9.30$ & & 0.326 \\
\hline Median(range) & $77(69-83)$ & $84(75-88)$ & & & $80(72-87)$ & $84(75-88)$ & & \\
\hline \multicolumn{9}{|l|}{$\begin{array}{l}\text { Apache II } \\
\text { score on ICU }\end{array}$} \\
\hline Mean \pm S.D. & $19.51 \pm 2.73$ & $19.65 \pm 3.60$ & & 0.849 & $20.00 \pm 2.73$ & $19.65 \pm 3.60$ & & 0.714 \\
\hline Median(range) & $19(18-22)$ & $20(17-22)$ & & & $20(17-22)$ & $20(17-22)$ & & \\
\hline \multicolumn{9}{|l|}{ SOFA score } \\
\hline Mean \pm S.D. & $13.15 \pm 2.41$ & $13.17 \pm 1.87$ & & 0.974 & $13.70 \pm 2.55$ & $13.17 \pm 1.87$ & & 0.307 \\
\hline Median(range) & $13[11-15]$ & $13[12-14]$ & & & $13[12-15]$ & $13[12-14]$ & & \\
\hline \multicolumn{9}{|l|}{ Albumin $(\mathrm{g} / \mathrm{L})$} \\
\hline Mean \pm S.D. & $27.86 \pm 5.50$ & $28.10 \pm 6.53$ & & 0.867 & $27.24 \pm 3.70$ & $28.10 \pm 6.53$ & & 0.587 \\
\hline Median(range) & $\begin{array}{c}28.0(25.7- \\
32.3)\end{array}$ & $\begin{array}{c}29.4(23.7- \\
33.5)\end{array}$ & & & $\begin{array}{c}27.7(25.7- \\
29.4)\end{array}$ & $\begin{array}{c}29.4(23.7- \\
33.5)\end{array}$ & & \\
\hline \multicolumn{9}{|l|}{$\begin{array}{l}\text { Serum } \\
\text { creatinine } \\
(\mu \mathrm{mol} / \mathrm{L})\end{array}$} \\
\hline Mean士S.D. & $62.73 \pm 29.81$ & $79.00 \pm 26.24$ & & $0.026^{*}$ & $81.09 \pm 32.46$ & $79.00 \pm 26.24$ & & 0.812 \\
\hline Median(range) & $50(42-77)$ & $76(63-89)$ & & & 74(53-105) & $76 \square 63-89 \square$ & & \\
\hline \multicolumn{9}{|l|}{$\begin{array}{l}\text { Clinical } \\
\text { Outcomes }\end{array}$} \\
\hline $\begin{array}{l}\text { Composite } \\
\text { outcome of } \\
\text { new-onset } \\
\text { AKI or RRT }\end{array}$ & $4(7.3)$ & $3(13.0)$ & 0.143 & 0.705 & $4(17.4)$ & $3(13.0)$ & 0.000 & 1.000 \\
\hline $\begin{array}{l}\text { Clinical } \\
\text { success rate, } n \\
(\%)\end{array}$ & $39(70.9)$ & $19(82.6)$ & 1.164 & 0.281 & $16(69.6)$ & 19(82.6) & 1.075 & 0.300 \\
\hline $\begin{array}{l}\text { 28-day all- } \\
\text { cause } \\
\text { mortality }\end{array}$ & 4 & 0 & 0.585 & 0.444 & $3(13.0)$ & $1(4.3)$ & 0.274 & 0.601 \\
\hline
\end{tabular}

$* p \llbracket 0.05$

Abbreviations: APACHE II, acute physiology and chronic health evaluation II; SOFA, Sequential Organ Failure Assessment $\rrbracket \mathrm{AKI}$, acute kidney injury; RRT, renal replacement therapy[

\section{Figures}


$-\mathrm{A}$

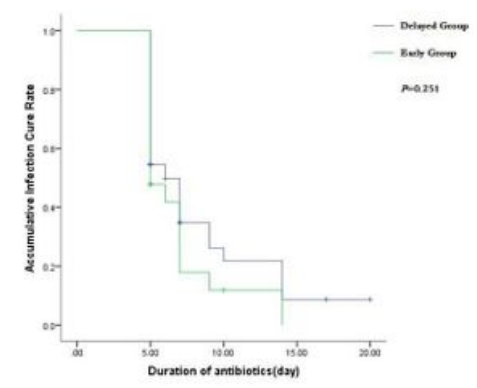

$-\mathrm{C}$

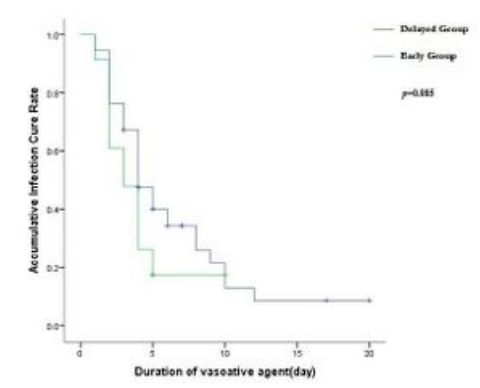

$-\mathrm{E}$

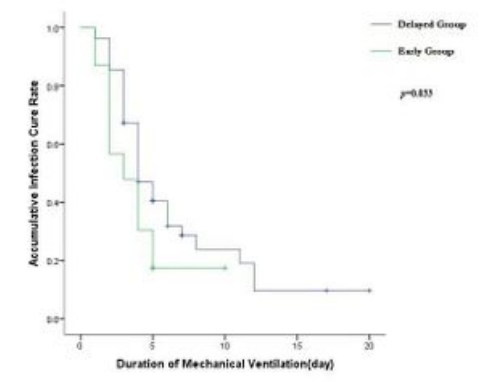

B

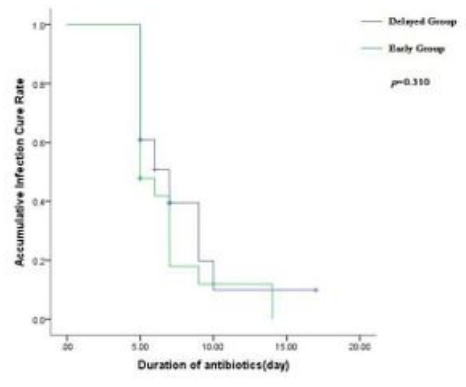

D

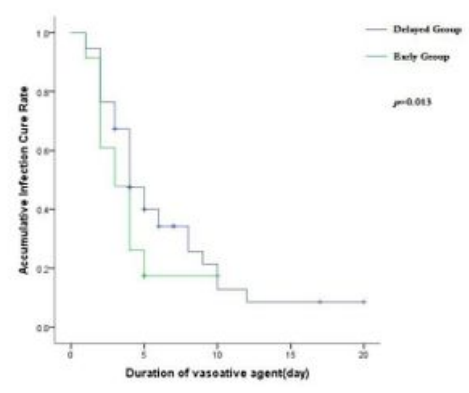

$\mathrm{F}$

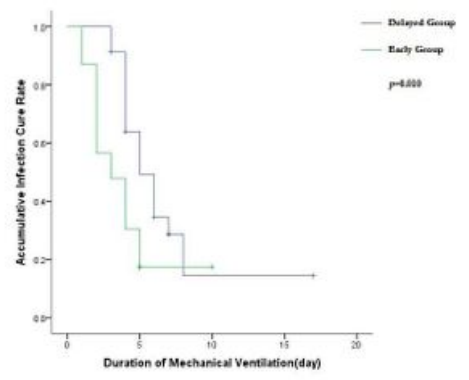

\section{Figure 1}

Kaplan-Meier curves for (A) Duration of Antibiotics between the two groups before weighting; (B) Duration of Antibiotics after weighting; (C) Duration of Vasoactive Agent between the two groups before weighting; (D) Duration of Vasoactive Agent after weighting; (E) Duration of Mechanical Ventilation between the two groups before weighting; (D) Duration of Mechanical Ventilation after weighting. 